\title{
Zum Ursprung der Herbergsuche
}

Niko Kuret

$1906-1995$

Die Herbergsuche oder das Frautragen, wie P. Sartori seinen Artikel im Handwörterbuch des deutschen Aberglaubens II $^{1}$ betitelt hat, ist ein Kind der Barockzeit. Der Ursprung des Brauches ist $\mathrm{m}$.W. noch nicht erörtert worden.

Die Angaben zeigen nach Spanien.

In einem Vortrag in der Sociedad folklorica de México im J. 1942 vertrat jedoch Germán Andrada Labastida ${ }^{2}$ die Meinung, der auch in Mexico verbreitete Brauch sei mexikanischen Ursprungs, entstanden in der Nähe der Pyramiden von Teotihuacan, im kleinen Dorf San Agustin Acolman. Die Azteken feierten tatsächlich den Geburtstag des Kriegsgottes Huizilopachtli in den Tagen des christlichen Weihnachtsfestes. Dies bewog die augustinischen Ordensbrüder, ähnliche Festlichkeiten zu Weihnachten einzuführen, darunter die neuntägige Reise Marias und des hl. Josef nach Bethlehem. Der Augustinerprior Diego de Soria aus dem Kloster von San Agustin Acolman erreichte bei Papst Sixtus V., daß eine neuntägige Andacht mit besonderen Ablässen bedacht wurde. Die Novene hätte den Brauch der Herbergsuche hervorgebracht. Er wurde das "pedir posada" genannt.

Spätere Autoren sind jedoch anderer Meinung. Die Herbergsuche soll in Spanien, in Andalusien, im 16. Jh. entstanden sein.

Im schicksalsschweren Jahr 1492 wurden die Mauren (Moriscos) aus Spanien verdrängt. Befreit wurden Granada, Malaga, Almeria, und ein großes Gebiet wartete auf die Missionierung. Die Missionstätigkeit übernahmen hauptsächlich die Franziskaner. Bei der Missionsarbeit bedienten sie sich aller Mittel, die von der Barockzeit hervorgebracht worden waren. Gefördert wurde besonders alles Theatralische. Man entdeckte das dramatische Motiv in der Herbergsuche, wo der Hauswirt das heilige Paar nicht aufnehmen wollte. Deshalb war die Entstehung eines eigenartigen Umgangs-“Spiels” möglich. Im selben Jahr aber entdeckte Kolumbus das Neue Spanien, welches nun auch der Missionierung erschlossen wurde; es waren hauptsächlich wieder Franziskaner, die die Missionsarbeit übernahmen. Sie brachten die Mittel mit, die sie im Süden Spaniens erfolgreich erprobt hatten. Das Spielerische wurde von den Einheimischen Neu Spaniens (Mexikos) mit Gefallen aufgenommen. ${ }^{3}$ Als Entstehungsort der mexikanischen Herbergsuche (las posadas) wird Ciudad México angeführt. Der in den Kirchen und in Klöstern begonnene Brauch zog bald in den

1 HDA II, Berlin und Leipzig 1929/1930, 1777-1778.

2 So Rafael Solano, Las posadas, in: Artes de México II, ed. Nacimiento, villancicoso, pastorales. México 1960, 10.

3 S. Torquato Luca de Tena, Posada y pastorales, in: Ztg. ABC, Madrid 26. dec. 1989 (und seine brieflichen Auskünfte vom 28. 8. 1989), sowie schon don Mariano de Carrer, Posibles origenes de las tipicas posadas mexicanas, in: Anuario de la Sociedad Folklorica de México II. México 1945. 
häuslichen Umkreis über. ${ }^{4}$ Hier wurde er nach Aztekenart von einer fröhlichen Unterhaltung (das Zerschlagen der pinata) umrahmt und von einem üppigen Schmaus begleitet.

Bei der mexikanischen Herbergsuche trägt ein Knabe die Statue des hl. Josef, ein Mädchen eine Marienstatue im Umzug. Es wird gesungen, ein Wechselgesang zwischen dem "Hauswirt" (hinter der geschlossenen Tür) und den Umgängern (vor der Tür). Die letzte, im Haus gesungene Strophe lautet:

Eres tu José, tu esposa es Maria,

entren peregrinos, no les conocía. ${ }^{5}$

Der Brauch ist (war) in ganz Mexiko (auch in New Mexico in den USA) verbreitet, kam auch nach Venezuela, ${ }^{6}$ ist jedoch im Absterben begriffen. Der religiöse Teil, der Wechselgesang, wird fortgelassen, es bleibt bloß die Unterhaltung. Wohl aber wird der Brauch auch als kirchliche Andacht abgehalten, wie ich sie im J. 1968 in Guadalajára (Jal.) erlebt habe.

Auch im heutigen Spanien ist der Brauch stark abgekommen. Man findet ihn bloß noch bei den Gebirglern der Sierra und der Estremadura.

Die Wege, auf denen der Brauch nach Mitteleuropa gewandert ist, sind im einzelnen noch unbekannt. Es geschah allerdings im Rahmen des einstigen großen Habsburgerreiches, in dem "die Sonne nie unterging”. Der Brauch wurde auffallenderweise von den katholischen Ländern deutscher Zunge und ihrer Nachbarschaft aufgenommen und lebt da fort. Sartori o.c. führt als Verbreitungsländer den Pinzgau, Baden und die Obersteiermark an. Über den Ursprung berichtet er nichts, seine Meinung, der Brauch erinnere an die germanische Nerthusfeier (Tac. Germ. 40), ist unhaltbar. Er soll als "notandus abusus" von der Geistlichkeit verboten worden sein. Daß ihm die Ära Josephs II nicht freundlich war, ist klar.

Den Brauch erwähnt vorübergehend Rudolf Berliner in seinem großen Werk: ${ }^{7}$ Die Münchner Jesuiten bezahlten 1736 den Maler, der das Bild "für die Reiss unser lieben Frauen und St. Joseph" gemalt (oder restauriert) hatte. Daraus ginge hervor, dass der Brauch in der ersten Hälfte des 18. Jahrh. in Bayern bekannt und von den Jesuiten (in München) gepflegt wurde. Man berichtet auch von einem handschriftlichen Büchlein aus dem Kloster der Franziskaner in Mindelheim und den Anweisungen und Gebeten für die Andacht "anno 1758”. Der Brauch soll von Anna Katharina, der Tochter Ferdinands, des Herzogs von Tirol, um 1600 in Hall eingeführt worden $\operatorname{sein} .^{8} \mathrm{Er}$ ist in deutschen Landen, in Bayern, Schwaben, Württemberg, Tirol und Salzburg anzutreffen.

Er ist auch zu den Slowenen gelangt - zu den Friauler Slowenen im heutigen Italien und zu den Slowenen in Steiermark und Krain. Der mündlichen Überlieferung nach soll er in Friaul schon Ende des 18. Jahrhunderts heimisch gewesen sein.

Der zum Brauch gehörige (spanische) Wechselgesang konnte allerdings in Mitteleuropa nicht übernommen werden. Bei den Friauler Slowenen und auch anderswo in Slowenien verläuft er auch ohne Wechselgesang. Es ist jedoch anzunehmen, daß schon bald ein dem spanischen entsprechender Wechselgesang (in deutscher Sprache) entstanden ist. Diese

4 S. Luis Rublúo, La navidad méxicana en el siglo XVI, in: Artes de México 19, no. 157, México 1973, 6 ff.

5 Du dürftest Josef sein, deine Gemahlin ist Maria, tretet ein, Pilgerpaar, wir haben euch erkannt.

${ }^{6}$ Nives de Hoyos Sancho, Nacimineto en Hispano-America, in: Revista espanola de indigenismo. Madrid 7, diciembre 1965/6, und briefliche Mitteilung vom 27. 8. 1985.

7 Rudolf Berliner, Die Weihnachtskrippe. München 1955, 23, Anm. 275.

8 Annie Hertinger, “Wer klopfet an?” Regensburg 1948. 
Fassung bleibt unbekannt, im 19. Jh. aber entstand das bekannte Dialoglied "Wer klopfet an?". Hartmann hat es in seine Sammlung mit Abels Melodieaufzeichnung eingereiht. ${ }^{9} \mathrm{Er}$ bezeichnet es als Volkslied "in Kiefersfelden, Salzburg, Wildschönau, auch in Oberbayern verbreitet". Wann das Lied entstanden ist, ist nicht festzustellen. Allein die Slowenen hatten sich eine slowenische Fassung davon besorgt und sie in einem Jahrbuch ("Drobtinice") im J. 1861 veröffentlicht. Das deutsche Original muß also vor 1861 entstanden und gedruckt worden sein. Interessanterweise führt die slowenische Übersetzung am Ende den Namen des Autors an. Es soll ein (in slowenischer Graphie) J. Šehel (Schechel ?) gewesen sein. Man dürfte es also nicht als "Volkslied" bezeichnen!

Der Brauch war bei den Slowenen schon abgekommen, wurde aber Ende des 19. Jahrh. wiederbelebt und ist auch heute in allen slowenischen Gebieten - ohne Ausnahmen - verbreitet.

Eine ähnliche Wiederbelebung des schon abgekommenen Brauches ist auch in Tirol zu verzeichnen.

Das Lied “Wer klopfet an?” öffnet allerdings neue Fragen. Es umfasst bloß sechs, in einigen Fassungen sogar nur vier Strophen: "beim ersten Haus”, "beim zweiten Haus” etc. ...” beim sechsten (vierten) Haus". Wenn das Lied einer Novene diente, wo bleiben die restlichen "Häuser" bis zum neunten? Oder singt man bei jedem Haus alle vorhandenen Strophen?

Befremdend ist weiters, daß die letzte Strophe keinen Übergang zum Empfang im Hause enthält, sondern bloß den Hinweis auf einen Stall in der Nähe, worauf die Umgänger ins Haus eintreten dürfen. Der Übergang ist im mexikanischen Liede unvergleichlich freundlicher: man bereut es, daß man das Heilige Paar nicht früher erkannt hat und läßt es mit Freuden ins Haus eintreten!

Die deutsche Fassung des Liedes "Wer klopfet an?" liegt in zahlreichen Drucken vor, so z.B. im Liederbuch Anton Dawidowicz "Komm, sing mit” Österr. Liederbuch (Innsbruck 1962, 192). Bei den Slowenen wird der slowenische Text auch handschriftlich überliefert.

Der eigenartige Brauch der Herbergsuche oder des Frautragens ist noch immer mangelhaft erforscht, weil Berichte davon nur spärlich sind oder überhaupt nicht vorliegen. Dieser kleine Beitrag dürfte weiteren Untersuchungen den Weg weisen.

\footnotetext{
9 August Hartmann, Volkslieder. In Bayern, Tirol und Land Salzburg gesammelt von -. Mit vielen Melodien aus dem Volksmunde aufgezeichnet von Hyazinth Abel. I. Volkstümliche Weihnachtslieder. Leipzig 1884, 121-124 (78. Melodie "Wer klopfet an?).
} 